\title{
PARTIAL REVELATION OF CERTIFIED IDENTITY
}

\author{
Fabrice Boudot \\ France Télécom $R \& D$ \\ 42 rue des Coutures, B.P. 6243 \\ 14066 Caen CEDEX 4, France \\ fabrice.boudot@francetelecom.fr
}

Abstract

We present in this paper an implementation of an electronic ID card which allows its holder to reveal (and prove) only a part of his identity.

In many cases, only a few pieces of information are required from the user, and classical schemes do not allow him to reveal only these specific pieces. For example, he must reveal his whole identity even though he is just required to prove that he is of age. To solve this problem, we present in this paper an identification scheme which allows the prover to reveal to the verifier only a part of his identity. Then, depending on the context, the user will be able to prove his full identity, only a part of it or just that he is of age. The part of the identification scheme performed by the smart card requires less than 10 exponentiations which can be pre-computed, so it can be easily implemented in a smart card.

\section{INTRODUCTION}

The set-up of an identification scheme is a tiresome process, as the third party which certifies the public key of each user must be convinced that this user is the person he claims to be. Up to now, the only way to prove indisputably one's identity to this third party is to go and see him with an ID card or a passport. It is desirable that this process would happen only once, so the resulting certificate must be usable in any case. But, depending on the context, the user may later on be required to give more or less information on his identity : in some cases, the full identity (name, address, date or birth, ... ) is asked for, when in other cases, the verifier only requires a proof that the user is of age. In the latter case, as 
the user is allowed to stay anonymous, we must allow him to prove that he is of age without disclosing his name or even his date of birth.

We present in this paper an implementation of an ID card on a smart card. This card contains her full identity : her name, her address, her date of birth, her place of birth and every element usually written in an ID card. It allows Alice to give her full identity to another entity (and prove that this identity is correct), or only a part of her identity, e.g. her name and her address without her date of birth. Moreover, she can prove that an element of her identity (typically her age) belongs to an interval, e.g. that she has reached her majority. She can also escrow a part of her identity: she will stay anonymous, but a judge will be able to retrieve her identity.

We briefly describe our model: Let $I$ be Alice's identity string which is the concatenation of several strings NAME, ADDR, DATE, PLACE and $O T H E R$ which contains her name, her address, her date of birth, her place of birth and other data about her. A certification authority, after being ensured that all these data objects are correct (e.g. by checking Alice's physical ID card) issues her with a signature $S$ of $I$. This signature $S$ is stored in a smart card.

In the first step, the smart card creates a commitment $F$ to Alice's identity string $I$ and proves to the verifier that it knows a signature $S$ of this committed number hidden in $F$. This convinces the verifier Bob that the ID card is valid and that the identity of the owner is hidden in $F$, but without providing him with any information about Alice's identity. Moreover, he is unable to establish a link between two "blind" identifications from the same user.

The second step can be performed by Alice's personal computer, if we allow this computer to know Alice's full identity. Alice creates five commitments to each data object NAME, ADDR,DATE PLACE and $O T H E R$ and proves to Bob that the concatenation of these committed numbers is $I$.

Finally, in the third step, each data object is processed separately : it can be hidden, revealed, escrowed or Alice can prove that it belongs to an interval.

If we let Alice's PC know her full identity, the main part of the scheme can be performed by this computer. The only part which must be performed by the smart card requires less than 10 exponentiations which can all be pre-computed. So, an identification can be performed in reasonable time by a smart card without cryptoprocessor if the exponentiatons are pre-computed, or otherwise by a smart card with cryptoprocessor.

Notations: Throughout this paper, $\mathbb{Z}_{n}$ denotes the residue class ring modulo $n$, and $\mathbb{Z}_{n}^{*}$ denotes the multiplicative group of invertible elements 
in $\mathbb{Z}_{n} . a \| b$ denotes the concatenation of the strings $a$ and $b$. We denote by $P K_{t}(x: \mathcal{R}(x))$ a zero-knowledge proof of knowledge of $x$ such that $\mathcal{R}(x)$ is true, where $t$ is a security parameter.

Organization of the paper: In Section 2, we present the basic tools we will use in our scheme. We describe our scheme in Section 3. Finally, we conclude in section 4 .

\section{BUILDING BLOCKS}

In this section, we present the building blocks we will use in our schemes. All these tools are already known and have been proven secure by their authors under (at least) the strong-RSA assumption introduced in [FO97] :

Strong RSA Assumption: There exist a probabilistic polynomial-time algorithm that on input $1^{|n|}$ outputs an RSA-modulus $n$ and an element $z \in \mathbb{Z}_{n}^{*}$ such that it is infeasible to find integers $e \notin\{-1,1\}$ and $u$ such that $z=u^{e} \bmod n$.

In this section, the building blocks are performed by two entities called Alice and Bob. We will only specify which kind of devices are used to perform these proofs (a smart card, a computer, a server, ... ) in the next section.

Throughout this section, $n$ is a large composite number which is the product of two strong primes $p=2 p^{\prime}+1$ and $q=2 q^{\prime}+1$ whose factorization is unknown by Alice, $g$ is an element of order $p^{\prime} q^{\prime}$ in $\mathbb{Z}_{n}^{*}$ and $h$ is an element of the group generated by $g$ such that both the discrete logarithm of $g$ in base $h$ and the discrete logarithm of $h$ in base $g$ are unknown by Alice. $H$ is a hash-function which outputs $2 t$-bit strings, where $t$ is a security parameter.

\subsection{THE FUJISAKI-OKAMOTO COMMITMENT SCHEME}

Let $s$ be a security parameter.

We denote by $E=E(x, r)=g^{x} h^{r} \bmod n$ a commitment to $x$ in base $(g, h)$, where $r$ is randomly selected over $\left\{-2^{s} n+1, \ldots, 2^{s} n-1\right\}$.

This commitment has first appeared in [FO97], and statistically reveals no information about the committed number $x$.

\subsection{SPECIAL PROOF THAT A COMMITTED NUMBER LIES IN A GIVEN RANGE}

Let $t, l$ and $s$ be three security parameters. A successful execution of this protocol convinces Bob that a committed number $x$ lies in 
$\left[-2^{t+l} b, 2^{t+l} b\right]$, but can be successfully executed by Alice with overwhelming probability only if $x$ lies in [0,b]. This proof is due to [CFT98].

Protocol: $\operatorname{SpecP} K_{t, l}\left(x, r: E=E(x, r) \wedge x \in\left[-2^{t+l} b, 2^{t+l} b\right]\right)$.

1 Alice picks random $\omega \in_{R}\left\{0, \ldots, 2^{t+l} b-1\right\}$ and $\eta \in_{R}\left\{0, \ldots, 2^{t+l+s} n\right.$ $-1\}$, and then computes $W=g^{\omega} h^{\eta} \bmod n$.

2 Then, she computes $C=H(W)$ and $c=C \bmod 2^{t}$.

3 Finally, she computes $D_{1}=\omega+x c$ and $D_{2}=\eta+r c$ (in $\mathbb{Z}$ ). If $D_{1} \in\left\{c b, \ldots, 2^{t+l} b-1\right\}$, she sends $\left(C, D_{1}, D_{2}\right)$ to Bob, otherwise she starts again the protocol.

4 Bob checks that $D_{1} \in\left\{c b, \ldots, 2^{t+l} b-1\right\}$ and that $C=H\left(g^{D_{1}} h^{D_{2}}\right.$ $\left.E^{-c}\right)$.

\subsection{PROOF THAT TWO COMMITMENTS HIDE THE SAME SECRET}

Let $t, l, s_{1}$ and $s_{2}$ be four security parameters.

Let $g_{1}$ be an element of large order in $\mathbb{Z}_{n}^{*}$ and $g_{2}, h_{1}, h_{2}$ be elements of the group generated by $g_{1}$ such that the discrete logarithm of $g_{1}$ in base $h_{1}$, the discrete logarithm of $h_{1}$ in base $g_{1}$, the discrete logarithm of $g_{2}$ in base $h_{2}$ and the discrete logarithm of $h_{2}$ in base $g_{2}$ are unknown by Alice.

We denote by $E_{1}\left(x, r_{1}\right)=g_{1}^{x} h_{1}^{r_{1}} \bmod n$ a commitment to $x$ in base $\left(g_{1}, h_{1}\right)$ where $r_{1}$ is randomly selected over $\left\{-2^{s_{1}} n+1, \ldots, 2^{s_{1}} n-1\right\}$, and $E_{2}\left(x, r_{2}\right)=g_{2}^{x} h_{2}^{r_{2}} \bmod n$ a commitment to $x$ in base $\left(g_{2}, h_{2}\right)$ where $r_{2}$ is randomly selected over $\left\{-2^{s_{2}} n+1, \ldots, 2^{s_{2}} n-1\right\}$.

Alice secretly holds $x \in\{0, \ldots, b\}$. Let $E=E_{1}\left(x, r_{1}\right)$ and $F=$ $E_{2}\left(x, r_{2}\right)$ be two commitments to $x$. She wants to prove to Bob that she knows $x, r_{1}, r_{2}$ such that $E=E_{1}\left(x, r_{1}\right)$ and $F=E_{2}\left(x, r_{2}\right)$, i.e. that $E$ and $F$ hide the same secret $x$.

This protocol is derived from proofs of equality of two discrete logarithms from [CEG87], [CP92] and [Bao98], combined with a proof of knowledge of a discrete logarithm modulo $n$ [Gir91].

Protocol: $P K_{t, l}\left(x, r_{1}, r_{2}: E=E_{1}\left(x, r_{1}\right) \wedge F=E_{2}\left(x, r_{2}\right)\right)$.

1 Alice picks random $\omega \in\left\{1, \ldots, 2^{l+t} b-1\right\}, \eta_{1} \in\left\{1, \ldots, 2^{l+t+s_{1}} n-\right.$ $1\}, \eta_{2} \in\left\{1, \ldots, 2^{l+t+s_{2}} n-1\right\}$. Then, she computes $W_{1}=g_{1}^{\omega} h_{1}^{\eta_{1}}$ $\bmod n$ and $W_{2}=g_{2}^{\omega} h_{2}^{\eta_{2}} \bmod n$.

2 Alice computes $c=H\left(W_{1} \| W_{2}\right)$. 
3 She computes $D=\omega+c x, D_{1}=\eta_{1}+c r_{1}, D_{2}=\eta_{2}+c r_{2}$ (in $\mathbb{Z}$ ) and sends $\left(c, D, D_{1}, D_{2}\right)$ to Bob.

4 Bob checks whether $c=H\left(g_{1}^{D} h_{1}^{D_{1}} E^{-c} \bmod n \| g_{2}^{D} h_{2}^{D_{2}} F^{-c} \bmod \right.$ $n)$.

\subsection{PROOF THAT A COMMITTED NUMBER IS A SQUARE}

Let $t, l$, and $s$ be three security parameters.

Alice secretly holds $x \in\{0, \ldots, b\}$. Let $E=E\left(x^{2}, r_{1}\right)$ be a commitment to the square of $x$ (in $\mathbb{Z}$ ). She wants to prove to Bob that she knows $x$ and $r_{1}$ such that $E=E\left(x^{2}, r_{1}\right)$, i.e. that $E$ hides the square $x^{2}$.

The first proof that a committed number is a square has appeared in [FO97].

Protocol: $P K_{t, l}\left(x, r_{1}: E=E\left(x^{2}, r_{1}\right)\right)$.

1 Alice picks random $r_{2} \in\left\{-2^{s} n+1, \ldots, 2^{s} n-1\right\}$ and computes $F=E\left(x, r_{2}\right)$.

2 Then, Alice computes $r_{3}=r_{1}-r_{2} x$ (in $\left.\mathbb{Z}\right)$. Note that $r_{3} \in\left\{-2^{s} b n+\right.$ $\left.1, \ldots, 2^{s} b n-1\right\}$. Then, $E=F^{x} h^{r_{3}} \bmod n$.

3 As $E$ is a commitment to $x$ in base $(F, h)$ and $F$ is a commitment to $x$ in base $(g, h)$, Alice can run $P K\left(x, r_{2}, r_{3}: F=g^{x} h^{r_{2}} \bmod n \wedge\right.$ $\left.E=F^{x} h^{r_{3}} \bmod n\right)$, the proof that two commitments hide the same secret described in section 2.3. She gets $\left(c, D, D_{1}, D_{2}\right)$.

4 She sends $\left(F, c, D, D_{1}, D_{2}\right)$ to Bob.

5 Bob checks that $P K\left(x, r_{2}, r_{3}: F=g^{x} h^{r_{2}} \bmod n \wedge E=F^{x} h^{r_{3}} \bmod \right.$ $n$ ) is valid.

\subsection{AN "EXACT” PROOF THAT A COMMITTED NUMBER LIES IN A GIVEN RANGE}

In this section, we recall results from [Bou00]. We first present a protocol which allows Alice to prove to Bob that a committed number $x \in[a, b]$ belongs to $[a-\theta, b+\theta]$, where $\theta=2^{t+l+1} \sqrt{b-a}$, and then present a slightly modified version of this first protocol which convinces Bob that $x$ belongs to $[a, b]$ and not a larger interval.

\subsubsection{First Proof.}


Let $t, l$ and $s$ be three security parameters. This protocol allows Alice to prove to Bob that a committed number $x \in[a, b]$ belongs to $[a-\theta, b+\theta]$, where $\theta=2^{t+l+1} \sqrt{b-a}$

Protocol: $P K(x, r: E=E(x, r) \wedge x \in[a-\theta, b+\theta])$.

1 Both Alice and Bob compute $\tilde{E}=E / g^{a} \bmod n$ and $\bar{E}=g^{b} / E \bmod$ $n$. Alice sets $\tilde{x}=x-a$ and $\bar{x}=b-x$. Now, Alice must prove to Bob that both $\tilde{E}$ and $\bar{E}$ hide secrets which are greater than $-\theta$.

2 Alice computes:

$$
\begin{aligned}
& \tilde{x}_{1}=\lfloor\sqrt{x-a}\rfloor, \tilde{x}_{2}=\tilde{x}-\tilde{x}_{1}^{2}, \\
& \bar{x}_{1}=\lfloor\sqrt{b-x}\rfloor, \bar{x}_{2}=\bar{x}-\bar{x}_{1}^{2} .
\end{aligned}
$$

Then, $\tilde{x}=\tilde{x}_{1}^{2}+\tilde{x}_{2}$ and $\bar{x}=\bar{x}_{1}^{2}+\bar{x}_{2}$, where $0 \leq \tilde{x}_{2} \leq 2 \sqrt{b-a}$ and $0 \leq \bar{x}_{2} \leq 2 \sqrt{b-a}$.

3 Alice randomly selects $\tilde{r}_{1}$ and $\tilde{r}_{2}$ in $\left\{-2^{s} n+1, \ldots, 2^{s} n-1\right\}$ such that $\tilde{r}_{1}+\tilde{r}_{2}=r$, and $\bar{r}_{1}$ and $\bar{r}_{2}$ such that $\bar{r}_{1}+\bar{r}_{2}=-r$.

4 Alice computes:

$$
\begin{aligned}
& \tilde{E}_{1}=E\left(\tilde{x}_{1}^{2}, \tilde{r}_{1}\right), \tilde{E}_{2}=E\left(\tilde{x}_{2}, \tilde{r}_{2}\right), \\
& \bar{E}_{1}=E\left(\bar{x}_{1}^{2}, \bar{r}_{1}\right), \bar{E}_{2}=E\left(\bar{x}_{2}, \bar{r}_{2}\right) .
\end{aligned}
$$

5 Alice sends $\tilde{E}_{1}$ and $\bar{E}_{1}$ to Bob. Bob computes $\tilde{E}_{2}=\tilde{E} / \tilde{E}_{1}$ and $\bar{E}_{2}=\bar{E} / \bar{E}_{1}$.

6 Alice executes with Bob

$$
\begin{aligned}
& P K_{t, l}\left(\tilde{x}_{1}, \tilde{r}_{1}: \tilde{E}_{1}=E\left(\tilde{x}_{1}^{2}, \tilde{r}_{1}\right)\right), \\
& P K_{t, l}\left(\bar{x}_{1}, \bar{r}_{1}: \bar{E}_{1}=E\left(\bar{x}_{1}^{2}, \bar{r}_{1}\right)\right)
\end{aligned}
$$

which prove that both $\tilde{E}_{1}$ and $\bar{E}_{1}$ hide a square.

7 Let $\theta=2^{t+l+1} \sqrt{b-a}$. Alice executes with Bob the two following special proofs from section 2.2 :

$$
\begin{aligned}
& S p e c P K_{t, l}\left(\tilde{x_{2}}, \tilde{r_{2}}: \tilde{E_{2}}=E\left(\tilde{x_{2}}, \tilde{r_{2}}\right) \wedge \tilde{x_{2}} \in[-\theta, \theta]\right), \\
& S p e c P K_{t, l}\left(\overline{x_{2}}, \overline{r_{2}}: \overline{E_{2}}=E\left(\overline{x_{2}}, \overline{r_{2}}\right) \wedge \overline{x_{2}} \in[-\theta, \theta]\right) .
\end{aligned}
$$

which prove that both $\tilde{E}_{2}$ and $\bar{E}_{2}$ hide numbers which belong to $[-\theta, \theta]$, where $\theta=2^{t+l+1} \sqrt{b-a}$, instead of proving that they belong to $[0,2 \sqrt{b-a}]$.

In this proof, it is shown that both $\tilde{x}$ and $\bar{x}$ are the sums of a square and a number which is greater than $-\theta$. As a square is always positive, Bob is convinced that $\tilde{x}=x-a>-\theta$ and that $\bar{x}=b-x>-\theta$, i.e. $x$ belongs to $[a-\theta, b+\theta]$. 


\subsubsection{Second Proof.}

By artificially enlarging the size of the secret, it is possible to convince Bob that a committed number $x \in[a, b]$ belongs to this interval and not a larger one.

First, Alice proves to Bob that she knows $x$, the committed value hidden in $E$.

Let $T=2 t+2 l+2+\left\lceil\log _{2}(b-a)\right\rceil$. We define $x^{\prime}=2^{T} x$, and $E^{\prime}=E^{2^{T}} . E^{\prime}$ is a Fujisaki-Okamoto commitment to $x^{\prime}$ that Alice can open. Moreover, as Alice knows how to open $E$, Bob is convinced that the committed value hidden in $E^{\prime}$ is $2^{T}$ times the committed value hidden in $E$.

As $x^{\prime} \in\left[2^{T} a, 2^{T} b\right]$, Alice can convince Bob that $x^{\prime} \in\left[2^{T} a-\theta, 2^{T} b+\theta\right]$, where $\theta=2^{t+l+1} \sqrt{2^{T} b-2^{T} a}$. As it is clear that $0<\theta<2^{T}$, Bob is convinced that $\left.x^{\prime} \in\right] 2^{T} a-2^{T}, 2^{T} b+2^{T}$.

Then, Bob is convinced that $x$, the committed value hidden in $E$, lies in the range $] a-1, b+1[$. Finally, as $x$ is an integer, Bob is convinced that $x \in[a, b]$.

\subsection{PROOF OF EQUALITY OF A COMMITTED THIRD ROOT AND A COMMITTED NUMBER}

This proof is derived from [FO98].

Let $N$ be a large composite number whose factorization is unknown by Alice. Alice secretly holds $x \in\{0, \ldots, b\}$. Let $E=E\left(x, r_{1}\right)$ and $F=$ $x^{3} \bmod N$ be a commitment to $x$ and a RSA encryption of $x$. She wants to prove to Bob that she knows $x$ and $r_{1}$ such that $E=E\left(x, r_{1}\right)$ and $F=E\left(x^{3} \bmod N, r_{2}\right)$.

Protocol: $P K_{t, l}\left(x, r_{1}, r_{2}: E=E_{1}\left(x, r_{1}\right) \wedge F=E\left(x^{3} \bmod N, r_{2}\right)\right.$.

1 Alice proves to Bob that the value committed by $E$ lies in $[0, N-1]$.

2 Alice computes $\alpha=\frac{\left(x^{3} \bmod N\right)-x^{3}}{N}$ (in $\left.\mathbb{Z}\right), G_{2}=E_{1}\left(x^{2}, r_{3}\right), G_{3}=$ $E_{1}\left(x^{3}, r_{4}\right)$ and $Z=E_{1}\left(\alpha N, r_{2}-r_{4}\right)$.

3 Alice proves to Bob that $E, G_{2}$ and $G_{3}$ are commitments to the same value respectively in bases $(g, h),(E, h)$ and $\left(G_{2}, h\right)$ using the building block presented in section 2.3 , and that she knows which value is committed by $Z$ in base $\left(g^{N}, h\right)$, using the building block presented in section 2.2 .

4 Bob checks these proofs, and also checks that $F=G_{3} Z \bmod n$. 


\section{SCHEME PROVIDING PARTIAL REVELATION OF IDENTITY}

\subsection{CERTIFICATION PROCEDURE}

The certification authority computes a large composite number $n$ which is the product of two strong primes $p=2 p^{\prime}+1$ and $q=2 q^{\prime}+1$. It defines an element $g$ of order $p^{\prime} q^{\prime}$ in $\mathbb{Z}_{n}^{*}$ and an element $h$ of the group generated by $g$. These values define the commitment scheme : $E(x, r)$, where $r$ is randomly selected over $\left[-2^{s} n, 2^{s} n\right]$, where $s$ is a security parameter.

Moreover, the certification authority computes a large RSA modulus $N$, and chooses a public exponent $e=3$ and a secret exponent $d$ such that $e d \equiv 1(\bmod \varphi(N))$.

When a user Alice wants to register to the system, she gives to the certification authority her name, her address, her date of birth, her place of birth and every element usually written in an ID card. She proves that she is honest by producing her physical ID card to the certification authority.

For each element of Alice's identity (name, address, ... ), the certification authority forms a string which contains this element and possibly some random characters so that these strings have the same length for every user. We call these strings : NAME, ADDR, DATE, PLACE, $O T H E R$, and their respective lengths : $L_{N A M E}, L_{A D D R}, L_{D A T E}$, $L_{P L A C E}$ and $L_{O T H E R}$.

Finally, Alice's identity string is the concatenation of these strings:

$$
I=N A M E\|A D D R\| D A T E\|P L A C E\| O T H E R
$$

We denote $L$ the length of the string $I$, and set $K=1+2^{L}+2^{2 L}+2^{3 L}$.

To certify this identity, the certification authority signs the ID string $I$ by computing:

$$
S=(K I)^{d}=(I\|I\| I \| I)^{d} \bmod N
$$

and gives $S$ to Alice which keeps this signature secret.

In the current state of the art, this signature scheme is secure against known signatures attacks. The best known attacks on this kind of signatures is found in [CNS99], but they are only chosen-message attacks. So, a coalition of fraudulent users cannot forge a signature of a valid message $M\|M\| M \| M$ using the signature of their own identity. We have chosen this kind of redundancy because it provides a secure signature scheme against known-message attacks and it is possible to design a zeroknowledge proof of knowledge of $S$ and $I$ such that $S=(K I)^{d} \bmod N$, but any redundancy function which has the same characteristics is also appropriate. 
The ID card of each user contains $S$ and $I$. The secret $S$ is protected by the tamper-resistance of the smart card.

\section{2. "BLIND” IDENTIFICATION}

Alice wants to prove that her ID card is valid and that she is the owner of this ID card, i.e. she holds $S$ such that $S^{3} \bmod n$ is in the form $I\|I\| I \| I$, but without revealing her identity, i.e. the string $I$.

For this, Alice's card creates two commitments: $E=E\left(S, r_{1}\right)$, a commitment to her secret certificate $S$ and $F=E\left(I, r_{2}\right)$, a commitment to the ID string $I$, where $r_{1}$ and $r_{2}$ are randomly selected over $\left[-2^{s} n, 2^{s} n\right]$.

We denote $G=F^{K}=E\left(K I, K r_{2}\right)$. Then, the card performs the following proof:

$$
P K\left(S, r_{1}, r_{2}: E=E\left(S, r_{1}\right) \wedge G=E\left(S^{3} \bmod N, r_{2}\right)\right)
$$

which convinces Bob that she knows a third root of the value hidden in $G$. Moreover, Alice proves with techniques of section 2.5 that the value hidden in $F$ belongs to $\left[1,2^{L-1}\right]:$ this convinces Bob that the value hidden in $G$ is of the form $M\|M\| M \| M$ and that $M \neq 0$. As the signature scheme used to certify the users' identities is secure against known signature attacks, Bob is convinced that $F$ is the commitment of Alice's identity, but has no information about Alice's identity.

The blind identification is partly performed by the smart card : the smart card performs the proof of knowledge of a third root of the value hidden in $G$. It requires 8 exponentiations which can be pre-computed (pre-computation of $h^{r_{1}}, h^{r_{2}}$ for the computation of $E$ and $G, h^{r_{3}}, h^{r_{4}}$ for the computation of the values $G_{2}$ and $G_{3}$ in the protocol presented in section 2.6 and four exponentiations for the proof of equality of three commitments, a generalization of the protocol presented in section 2.3). The second part of the blind identification (i.e. the proof that the value hidden in $F$ belongs to $\left.\left[1,2^{L-1}\right]\right)$ can be performed by Alice's computer, if we let it know Alice's full identity.

\subsection{SEPARATION OF DATA OBJECTS}

Now, Bob is convinced that $F=E\left(I, r_{2}\right)$ is a commitment to Alice's identity. As Alice does not want to reveal her full identity but only some parts of it, she commits herself to each part of her identity by computing:

$$
\begin{aligned}
& F_{N A M E}=E\left(N A M E, r_{5}\right) \\
& F_{A D D R}=E\left(A D D R, r_{6}\right) \\
& F_{D A T E}=E\left(D A T E, r_{7}\right) \\
& F_{P L A C E}=E\left(P L A C E, r_{8}\right) \\
& F_{O T H E R}=E\left(O T H E R, r_{9}\right)
\end{aligned}
$$


where $r_{5}, r_{6}, r_{7}, r_{8}$ and $r_{9}$ are randomly selected over $\left[-2^{s} n, 2^{s} n\right]$, and proves to Bob that she knows which values are hidden in these five commitments, and that these values have the required length:

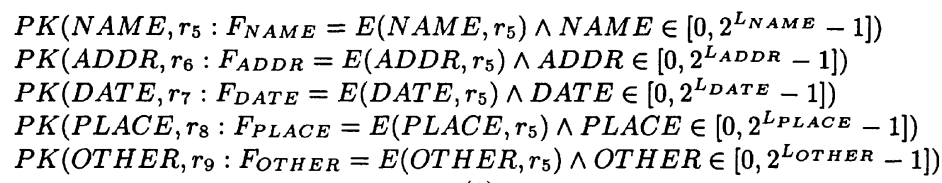

We denote $L_{1}=L_{A D D R}+L_{D A T E}+L_{P L A C E}+L_{O T H E R}, L_{2}=L_{D A T E}+$ $L_{P L A C E}+L_{O T H E R}, L_{3}=L_{P L A C E}+L_{O T H E R}$ and $L_{4}=L_{O T H E R}$, and $N A M E^{\prime}=N A M E \times 2^{L_{1}}, A D D R^{\prime}=A D D R \times 2^{L_{2}}, D A T E^{\prime}=D A T E \times$ $2^{L_{3}}$ and $P L A C E^{\prime}=P L A C E \times 2^{L_{4}}$.

Then, we have:

$\begin{array}{ccccccccccc}I & = & N A M E & \| & A D D R & \| & D A T E & \| & P L A C E & \| & O T H E R \\ N A M E^{\prime} & = & N A M E & \| \ldots 0 & \| & 0 \ldots 0 & \| & 0 \ldots 0 & \| & 0 \ldots 0 \\ A D D R^{\prime} & = & 0 \ldots 0 & \| & A D D R & \| & 0 \ldots 0 & \| \ldots 0 & \| & 0 \ldots 0 \\ D A T E^{\prime} & = & 0 \ldots 0 & \| & 0 \ldots 0 & \| & D A T E & \| \ldots 0 & 0 \ldots 0 & 0 \ldots 0 \\ P L A C E^{\prime} & = & 0 \ldots 0 & \| & 0 \ldots 0 & \| & 0 \ldots 0 & \| & P L A C E & \| & 0 \ldots 0 \\ O T H E R & = & 0 \ldots 0 & \| & 0 \ldots 0 & \| & 0 \ldots 0 & \| & 0 \ldots 0 & \| & O T H E R\end{array}$

Alice commits herself to the following commitments :

$$
\begin{aligned}
& G_{N A M E}=E\left(N A M E^{\prime}, r_{10}\right) \\
& G_{A D D R}=E\left(A D D R^{\prime}, r_{11}\right) \\
& G_{D A T E}=E\left(D A T E^{\prime}, r_{12}\right) \\
& G_{P L A C E}=E\left(P L A C E^{\prime}, r_{13}\right)
\end{aligned}
$$

where $r_{10}, r_{11}, r_{12}$ and $r_{13}$ are randomly selected over $\left[-2^{s} n, 2^{s} n\right]$ and such that $r_{10}+r_{11}+r_{12}+r_{13}+r_{9}=r_{2}$.

She sends these values to Bob which checks that:

$$
G_{N A M E} \times G_{A D D R} \times G_{D A T E} \times G_{P L A C E} \times F_{O T H E R} \equiv F(\bmod n)
$$

Finally, he performs the following proofs :

$$
\begin{aligned}
& P K\left(N A M E^{\prime}, r_{5}^{\prime}, r_{10}: F_{N A M E}^{L_{1}}=E\left(N A M E^{\prime}, r_{5}^{\prime}\right) \wedge G_{N A M E}=E\left(N A M E^{\prime}, r_{10}\right)\right) \\
& P K\left(A D D R^{\prime}, r_{6}^{\prime}, r_{11}: F_{A D D R}^{L_{2}}=E\left(A D D R^{\prime}, r_{6}^{\prime}\right) \wedge G_{A D D R}=E\left(A D D R^{\prime}, r_{11}\right)\right) \\
& P K\left(D A T E^{\prime}, r_{7}^{\prime}, r_{12}: F_{D A T E}^{L_{3}}=E\left(D A T E^{\prime}, r_{7}^{\prime}\right) \wedge G_{D A T E}=E\left(D A T E^{\prime}, r_{12}\right)\right) \\
& P K\left(P L A C E^{\prime}, r_{8}^{\prime}, r_{13}: F_{P L A C E}^{L_{4}}=E\left(P L A C E^{\prime}, r_{8}^{\prime}\right) \wedge G_{P L A C E}=E\left(P L A C E^{\prime}, r_{13}\right)\right)
\end{aligned}
$$

The proofs of knowledge (1) and the proofs of equalities (3) convince Bob that the string $N A M E^{\prime}$ ends by $L_{1}$ zeroes, the string $A D D R^{\prime}$ by $L_{2}$ zeroes, the string $D A T E$ by $L_{3}$ zeroes and the string $P L A C E^{\prime}$ by $L_{4}$ 
zeroes : as Alice can only know a single committed value in $F_{N A M E^{\prime}}$, there is an equality between the number $N A M E^{\prime}$ involved in the proofs of equalities in (3) and $2^{L_{1}} \times N A M E$, where $N A M E$ is the number involved in the proof of knowledge (1), i.e. the value committed by $F_{N A M E}$.

Moreover, as the proofs (1) are also proofs of membership to an interval, they convince Bob that the length of these strings are correct. So, Bob is convinced that the string $N A M E$ is at the right place in the string $N A M E^{\prime}$ and surrounded with zeroes, and so are $A D D R^{\prime}, D A T E^{\prime}$, $P L A C E^{\prime}$ and $O T H E R$.

Finally, the equality (2) convinces Bob that Alice's identity $I$ is the sum of $N A M E^{\prime}, A D D R^{\prime}, D A T E^{\prime}, P L A C E^{\prime}$ and $O T H E R$. As all these strings have the right format and contain the strings $N A M E, A D D R$, $D A T E, P L A C E$ and $O T H E R$ committed by $F_{N A M E}, F_{A D D R}, F_{D A T E}$, $F_{P L A C E}$ and $F_{O T H E R}$, Bob is convinced that these five commitments are commitments to Alice's name, Alice's address, Alice's date of birth, Alice's place of birth, and other data about Alice.

If we let a computer (e.g. Alice's PC) learn Alice's full identity, this part can be performed by this computer, as it does not require the knowledge of the secret $S$.

\subsection{PROCESSING OF EACH DATA OBJECT}

Once each data object (name, address, ... ) is isolated in a commitment, it can be processed in different ways : this data object can be kept secret, revealed, escrowed or Alice can prove that it belongs to an interval. We described here each possible process for a commitment $F=E(I N F O, r)$, where $I N F O$ is one of the strings $N A M E, A D D R$, $D A T E, P L A C E$ or OTHER.

Secrecy: Alice sends no information to Bob.

Revelation: Alice sends $I N F O$ and $r$ to Bob.

Bob checks that $E(I N F O, r)=F$.

Membership to an interval: Alice proves to Bob that the committed number hidden in $F$ belongs to an interval $[a, b]$. This process is very useful to convince Bob that Alice is young enough or old enough to access to a service.

Escrow: Alice performs a publicly verifiable secret sharing (see e.g. [Sta96], [YY98], [FO98] or [BT99]) of the secret INFO. Alice encrypts shares of her secret $I N F O$ which can be deciphered by some escrow authorities which can retrieve the secret INFO if they collaborate. Bob is able to check that Alice has encrypted the shares honestly. This leads to a revocable anonymity scheme. 
Once again, if we let a computer learn Alice's full identity, this part can be performed by this computer,

\section{CONCLUSION}

We have presented in this paper a reasonably efficient protocol which allows to reveal a part of one's identity to another entity and convince it that this identity is correct. This protocol can lead to an implementation on smart cards, as only less than 10 exponentiations (which can be precomputed) are required to perform the "blind" identification part, the only step which involves the smart card.

\section{Acknowledgments}

The author wishes to thank Marc Girault for helpful discussions and comments.

\section{References}

[Bao98] F. Bao, An Efficient Verifiable Encryption Scheme for Encryption of Discrete Logarithms, Proceedings of CARDIS'98, 1998.

[Bou00] F. Boudot, Efficient Proofs that a Committed Number Lies in an Interval, Proceedings of EUROCRYPT'2000, LNCS 1807, pp. 431-444, 2000.

[BT99] F. Boudot and J. Traoré, Efficient Publicly Verifiable Secret Sharing Schemes with Fast or Delayed Recovery, Proceedings of the International Conference on Information and Communication Security 1999, LNCS 1726, pp. 87102, 1999.

[CEG87] D. Chaum, J.-H. Evertse and J. van de Graaf, An Improved Protocol for Demonstrating Possession of Discrete Logarithm and Some Generalizations, Proceedings of EUROCRYPT'87, LNCS 304, pp. 127-141, 1988.

[CFT98] A. Chan, Y. Frankel and Y. Tsiounis, Easy Come Easy Go Divisible Cash, Proceedings of EUROCRYPT'98, LNCS 1403, pp. 561-575, 1998.

[CNS99] J.S. Coron, D. Naccache and J.P. Stern, On the Security of RSA Padding, Proceedings of CRYPTO'99, LNCS 1666, pp. 1-18, 1999.

[CP92] D. Chaum and T.P. Pedersen, Wallet Databases with Observers, Proceedings of CRYPTO'92, LNCS 740, pp. 89$105,1992$. 
[FO97] E. Fujisaki and T. Okamoto, Statistical Zero Knowledge Protocols to Prove Modular Polynomial Relations, Proceedings of CRYPTO'97, LNCS 1294, pp. 16-30, 1997.

[FO98] E. Fujisaki and T. Okamoto, A Practical and Provably Secure Scheme for Publicly Verifiable Secret Sharing and Its Applications, Proceedings of EUROCRYPT'98, LNCS 1403, pp. 32-46, 1998.

[Gir91] M. Girault, Self-Certified Public Keys, Proceedings of EUROCRYPT'91, LNCS 547, pp. 490-497, 1991.

[Sta96] M. Stadler, Publicly Verifiable Secret Sharing, Proceedings of EUROCRYPT'96, LNCS 1070, pp. 190-199, 1996.

[YY98] A. Young and M. Yung, Auto-Recoverable AutoCertifiable Cryptosystems, Proceedings of EUROCRYPT'98, LNCS 1403, pp. 17-31, 1998. 